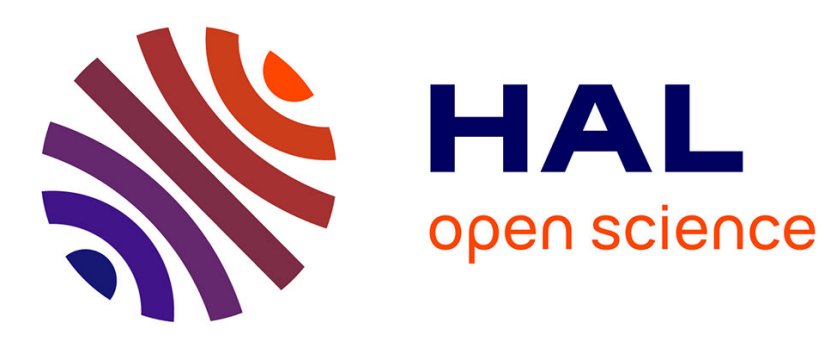

\title{
Differential Geometry Revisited by Biquaternion Clifford Algebra
}

\author{
Patrick R. Girard, Patrick Clarysse, Romaric Pujol, Liang Wang, Philippe \\ Delachartre
}

\section{- To cite this version:}

Patrick R. Girard, Patrick Clarysse, Romaric Pujol, Liang Wang, Philippe Delachartre. Differential Geometry Revisited by Biquaternion Clifford Algebra. Lecture Notes in Computer Science Curves and Surfaces, 9213, pp.216-242, 2015, 978-3-319-22804-4. 10.1007/978-3-319-22804-4_17. hal-01622682

\section{HAL Id: hal-01622682 \\ https://hal.science/hal-01622682}

Submitted on 24 Oct 2017

HAL is a multi-disciplinary open access archive for the deposit and dissemination of scientific research documents, whether they are published or not. The documents may come from teaching and research institutions in France or abroad, or from public or private research centers.
L'archive ouverte pluridisciplinaire HAL, est destinée au dépôt et à la diffusion de documents scientifiques de niveau recherche, publiés ou non, émanant des établissements d'enseignement et de recherche français ou étrangers, des laboratoires publics ou privés. 


\title{
DIFFERENTIAL GEOMETRY REVISITED BY BIQUATERNION CLIFFORD ALGEBRA
}

\author{
Patrick R. Girard ${ }^{1}$, Patrick Clarysse ${ }^{1}$, Romaric Pujol ${ }^{2}$, Liang Wang ${ }^{1}$, and \\ Philippe Delachartre ${ }^{1}$ \\ 1 Université de Lyon, CREATIS; CNRS UMR 5220; Inserm U1044; INSA-Lyon; \\ Université LYON 1, France, Bât. Blaise Pascal, 7 avenue Jean Capelle, F-69621 \\ Villeurbanne, France \\ 2 Université de Lyon, Pôle de Mathématiques, INSA-Lyon, Bât. Léonard de Vinci, 21 \\ avenue Jean Capelle, F-69621 Villeurbanne, France
}

\begin{abstract}
In the last century, differential geometry has been expressed within various calculi: vectors, tensors, spinors, exterior differential forms and recently Clifford algebras. Clifford algebras yield an excellent representation of the rotation group and of the Lorentz group which are the cornerstones of the theory of moving frames. Though Clifford algebras are all related to quaternions via the Clifford theorem, a biquaternion formulation of differential geometry does not seem to have been formulated so far. The paper develops, in $3 D$ Euclidean space, a biquaternion calculus, having an associative exterior product, and applies it to differential geometry. The formalism being new, the approach is intended to be pedagogical. Since the methods of Clifford algebras are similar in other dimensions, it is hoped that the paper might open new perspectives for a $4 \mathrm{D}$ hyperbolic differential geometry. All the calculi presented here can easily be implemented algebraically on Mathematica and numerically on Matlab. Examples, matrix representations, and a Mathematica work-sheet are provided.
\end{abstract}

Keywords: Clifford algebras, quaternions, biquaternions, differential geometry, rotation group $S O(3)$, hyperquaternion algebra

\section{Introduction}

Much of differential geometry is still formulated today within the $3 D$ vector calculus which was developed at the end of the nineteenth century. In recent years, new mathematical tools have appeared, based on Clifford algebras [110] which give an excellent representation of groups, such as the rotation group $S O(3)$ or the Lorentz group, which are the cornerstones of the theory of moving frames. Since the methods of Clifford algebras can easily be transposed to other dimensions, the question naturally arises of whether it is possible to rewrite differential geometry within a Clifford algebra in order to open new perspectives for $4 D$ modeling. Such an extension might proceed as follows. A $4 D$ tetraquaternion calculus has already been presented in [7,8]. A moving surface $O M=f(t, u, v)$ 
can be viewed as a hypersurface (with normal $n$ ) in a $4 D$ pseudo-euclidean space. The invariants are then obtained by diagonalizing the second fundamental form via a rotation around $n$ combined with a Lorentz boost along $n$, generalizing the methods presented here. Though Clifford algebras can be presented in various ways, the originality of the paper lies in the use biquaternions. We shall first introduce quaternions and Clifford algebras together with a demonstration of Clifford's theorem relating Clifford algebras to quaternions. Then, we shall develop the biquaternion calculus (with its associative exterior product) and show how classical differential geometry can be reformulated within this new algebraic framework.

\section{Clifford algebras: historical perspective}

\subsection{Hamilton's quaternions and biquaternions}

In 1843, W. R. Hamilton (1805-1865) discovered quaternions [11-17] which are a set of four real numbers:

$$
\begin{aligned}
a & =a_{0}+a_{1} i+a_{2} j+a_{3} k \\
& =\left(a_{0}, a_{1}, a_{2}, a_{3}\right) \\
& =\left(a_{0}, \vec{a}\right)
\end{aligned}
$$

where $i, j, k$ multiply according to the rules

$$
\begin{aligned}
i^{2} & =j^{2}=k^{2}=i j k=-1 \\
i j & =-j i=k \\
j k & =-k j=i \\
k i & =-i k=j .
\end{aligned}
$$

The conjugate of a quaternion is given by

$$
a_{c}=a_{0}-a_{1} i-a_{2} j-a_{3} k .
$$

Hamilton was to give a $3 D$ interpretation of quaternions; he named $a_{0}$ the scalar part and $\vec{a}$ the vector part. The product of two quaternions $a$ and $b$ is defined by

$$
\begin{aligned}
a b & =\left(a_{0} b_{0}-a_{1} b_{1}-a_{2} b_{2}-a_{3} b_{3}\right) \\
& +\left(a_{0} b_{1}+a_{1} b_{0}+a_{2} b_{3}-a_{3} b_{2}\right) i \\
& +\left(a_{0} b_{2}+a_{2} b_{0}+a_{3} b_{1}-a_{1} b_{3}\right) j \\
& +\left(a_{0} b_{3}+a_{3} b_{0}+a_{1} b_{2}-a_{2} b_{1}\right) k
\end{aligned}
$$

and in a more condensed form

$$
a b=\left(a_{0} b_{0}-\vec{a} \cdot \vec{b}, a_{0} \vec{b}+b_{0} \vec{a}+\vec{a} \times \vec{b}\right)
$$


where $\vec{a} \cdot \vec{b}$ and $\vec{a} \times \vec{b}$ are respectively the usual scalar and vector products. Quaternions (denoted by $\mathbb{H}$ ) constitute a non commutative field without zero divisors (i.e. $a b=0$ implies $a$ or $b=0$ ). At the end of the nineteenth century, the classical vector calculus was obtained by taking $a_{0}=b_{0}=0$ and by separating the dot and vector products. Hamilton also introduced complex quaternions he called biquaternions which we shall use in the next parts.

\subsection{Clifford algebras and theorem}

About the same time Hamilton discovered the quaternions, H. G. Grassmann (1809-1877) had the fundamental idea of a calculus composed of $n$ generators $e_{1}, e_{2}, \ldots e_{n}$ multiplying according to the rule $e_{i} e_{j}=-e_{j} e_{i}(i \neq j)$ [18-21]. In 1878 , W. K. Clifford (1845-1878) was to give a precise algebraic formulation thereof and proved the Clifford theorem relating Clifford algebras to quaternions. Though Clifford did not claim any particular originality, his name was to become attached to these algebras[22,23].

Definition 1. Clifford's algebra $C_{n}$ is defined as an algebra (over $\mathbb{R}$ ) composed of $n$ generators $e_{1}, e_{2}, \ldots, e_{n}$ multiplying according to the rule $e_{i} e_{j}=-e_{j} e_{i}(i \neq j)$ and such that $e_{i}^{2}= \pm 1$. The algebra $C_{n}$ contains $2^{n}$ elements constituted by the $n$ generators, the various products $e_{i} e_{j}, e_{i} e_{j} e_{k}, \ldots$ and the unit element 1 .

Examples of Clifford algebras (over $\mathbb{R}$ ) are

1. complex numbers $\mathbb{C}\left(e_{1}=i, e_{1}^{2}=-1\right)$.

2. quaternions $\mathbb{H}\left(e_{1}=i, e_{2}=j, e_{i}^{2}=-1\right)$.

3. biquaternions $\mathbb{H} \otimes \mathbb{C}\left(e_{1}=I i, e_{2}=I j, e_{3}=I k, I^{2}=-1, e_{i}^{2}=1, I\right.$ commuting with $i, j, k)$. Matrix representations of biquaternions are given in the appendix.

4. tetraquaternions $\mathbb{H} \otimes \mathbb{H}\left(e_{0}=j, e_{1}=k I, e_{2}=k J, e_{3}=k K, e_{0}^{2}=-1, e_{1}^{2}=\right.$ $e_{2}^{2}=e_{3}^{2}=1$, where the small $i, j, k$ commute with the capital $\left.I, J, K\right)[7,8]$.

All Clifford algebras are related to quaternions via the following theorem.

Theorem 1. If $n=2 m$ ( $m$ : integer), the Clifford algebra $C_{2 m}$ is the tensor product of $m$ quaternion algebras. If $n=2 m-1$, the Clifford algebra $C_{2 m-1}$ is the tensor product of $m-1$ quaternion algebras and the algebra $(1, \omega)$ where $\omega$ is the product of the $2 m$ generators $\left(\omega=e_{1} e_{2} \ldots e_{2 m}\right)$ of the algebra $C_{2 m}$.

Proof. The above examples of Clifford algebras prove the Clifford theorem up to $n=4$. For any $n$, Clifford's theorem can be proved by recurrence as follows $[24$, p. 378]. The theorem being true for $n=(2,4)$, suppose that the theorem is true for $C_{2(n-1)}$, to $C_{2(n-1)}$ one adds the quantities

$$
f=e_{1} e_{2} \ldots e_{2(n-1)} e_{2 n-1}, g=e_{1} e_{2} \ldots e_{2(n-1)} e_{2 n}
$$

which anticommute among themselves and commute with the elements of $C_{2(n-1)}$; hence, they constitute a quaternionic system which commutes with $C_{2(n-1)}$. From the various products between $f, g$ and the elements of $C_{2(n-1)}$ one obtains a basis of $C_{2 n}$ which proves the theorem. 
Hence, Clifford algebras can be formulated as hyperquaternion algebras the latter being defined as either a tensor product of quaternion algebras or a subalgebra thereof.

\section{Biquaternion Clifford algebra}

\subsection{Definition}

The algebra (over $\mathbb{R}$ ) has three anticommuting generators $e_{1}=I i, e_{2}=I j, e_{3}=$ $I k$ with $\mathrm{e}_{1}^{2}=e_{2}^{2}=e_{3}^{2}=1\left(I^{2}=-1, I\right.$ commuting with $\left.i, j, k\right)$. A complete basis of the algebra is given in the following table

$$
\begin{array}{|l|l|l|l|}
\hline 1 & i=e_{3} e_{2} & j=e_{1} e_{3} & k=e_{2} e_{1} \\
\hline I=e_{1} e_{2} e_{3} & I i=e_{1} & I j=e_{2} & I k=e_{3} \\
\hline
\end{array}
$$

A general element of the algebra can be written

$$
A=p+I q
$$

where $p=p_{0}+p_{1} i+p_{2} j+p_{3} k$ and $q=q_{0}+q_{1} i+q_{2} j+q_{3} k$ are quaternions. The Clifford algebra contains scalars $p_{0}$, vectors $I\left(0, q_{1}, q_{2}, q_{3}\right)$, bivectors $\left(0, p_{1}, p_{2}, p_{3}\right)$ and trivectors (pseudo-scalars) $I q_{0}$ where all coefficients $\left(p_{i}, q_{i}\right)$ are real numbers; we shall call these multivector spaces respectively $V_{0}, V_{1}, V_{2}$ and $V_{3}$. The product of two biquaternions $A=p+I q$ and $B=p^{\prime}+I q^{\prime}$ is defined by

$$
A B=\left(p p^{\prime}-q q^{\prime}\right)+I\left(p q^{\prime}+q p^{\prime}\right)
$$

where the products in parentheses are quaternion products. The conjugate of $A$ is defined as

$$
A_{c}=\left(p_{c}+I q_{c}\right)
$$

with $p_{c}$ and $q_{c}$ being the quaternion conjugates with $(A B)_{c}=B_{c} A_{c}$. The dual of $A$ noted $A^{*}$ is defined by

$$
A^{*}=I A
$$

and the commutator of two Clifford numbers by

$$
[A, B]=\frac{1}{2}(A B-B A) .
$$

\subsection{Interior and exterior products}

Products between vectors and multivectors In this section we shall adopt the general approach used in [4] though our algebra differs as well as several formulas. The product of two general elements of the algebra being given, one can define interior and exterior products of two vectors $a\left(=a_{1} i I+a_{2} j I+a_{3} k I\right)$ and $b$ via the obvious identity

$$
\begin{aligned}
a b & =\frac{1}{2}(a b+b a)-\left[-\frac{1}{2}(a b-b a)\right] \\
& =a \cdot b-a \wedge b
\end{aligned}
$$


with $a . b$ being the interior product

$$
\begin{aligned}
a . b & =\frac{1}{2}(a b+b a) \\
& =a_{1} b_{1}+a_{2} b_{2}+a_{3} b_{3} \in V_{0}
\end{aligned}
$$

and $a \wedge b$ the exterior product

$$
\begin{aligned}
a \wedge b & =-\frac{1}{2}(a b-b a) \\
& =\left(a_{2} b_{3}-a_{3} b_{2}\right) i+\left(a_{3} b_{1}-a_{1} b_{3}\right) j+\left(a_{1} b_{2}-a_{2} b_{1}\right) k \in V_{2}
\end{aligned}
$$

which has the same components as the pseudo-vector $\vec{a} \times \vec{b}$. Next we define the interior products $a . A_{p}$ and $A_{p} . a$ (with $2 \leq p \leq 3$ and $A_{p}=v_{1} \wedge v_{2} \wedge \ldots \wedge v_{p}$, $\left.v_{i} \in V_{1}\right)$

$$
a . A_{p}=\Sigma_{k=1}^{p}(-1)^{k}\left(a . v_{k}\right) v_{1} \wedge \ldots \wedge v_{k-1} \wedge v_{k+1} \wedge \ldots \wedge v_{p}
$$

together with

$$
A_{p} \cdot a \equiv(-1)^{p-1} a \cdot A_{p}
$$

Explicitly, we have

$$
\begin{gathered}
a \cdot\left(v_{1} \wedge v_{2}\right)=-\left(a \cdot v_{1}\right) v_{2}+\left(a \cdot v_{2}\right) v_{1} \\
a .\left(v_{1} \wedge v_{2} \wedge v_{3}\right)=-\left(a \cdot v_{1}\right)\left(v_{2} \wedge v_{3}\right)+\left(a \cdot v_{2}\right)\left(v_{1} \wedge v_{3}\right)-\left(a \cdot v_{3}\right)\left(v_{1} \wedge v_{2}\right) .
\end{gathered}
$$

The interior product $a . A_{p}$ allows the definition of the multivector $a \wedge A_{p}$ and $A_{p} \wedge a$ via the relations

$$
\begin{aligned}
& a A_{p}=a \cdot A_{p}-a \wedge A_{p} \\
& A_{p} a=A_{p} \cdot a-A_{p} \wedge a
\end{aligned}
$$

with

$$
A_{p} \wedge a=(-1)^{p} a \wedge A_{p} .
$$

Multiplying both sides of Eq. (29) with $(-1)^{p}$ and applying Eqs. $(25,30)$, we obtain

$$
(-1)^{p} A_{p} a=-a \cdot A_{p}-a \wedge A_{p}
$$

Combining Eqs. $(28,31)$, we obtain the formulas valid in all cases $(1 \leq p \leq 3)$

$$
\begin{gathered}
a . A_{p}=\frac{1}{2}\left[a A_{p}-(-1)^{p} A_{p} a\right] \in V_{p-1} \\
a \wedge A_{p}=-\frac{1}{2}\left[a A_{p}+(-1)^{p} A_{p} a\right] \in V_{p+1}
\end{gathered}
$$

$A_{3}$ being a pseudo-scalar, commuting with any Clifford number, we have in particular $a \wedge A_{3}=0$. 
Table 1. Interior and exterior products with their corresponding expressions in the classical vector calculus (with $B=b \wedge c, B_{1}=a \wedge b, B_{2}=c \wedge d, T_{1}=a \wedge b \wedge c, T_{2}=f \wedge g \wedge h$ and $\left.a, b, c, d, e, f, g, h \in V_{1}, T \in V_{3}\right)$

\begin{tabular}{ll}
\hline Multivector calculus & Classical vector calculus \\
\hline$a \cdot b=\frac{1}{2}(a b+b a) \in V_{0}$ & $\vec{a} \cdot \vec{b}$ \\
$a \wedge b=-\frac{1}{2}(a b-b a) \in V_{2}$ & $\vec{a} \times \vec{b}$ \\
$a \cdot B=\frac{1}{2}(a B-B a) \in V_{1}$ & $\vec{a} \times(\vec{b} \times \vec{c})$ \\
$a \wedge B=-\frac{1}{2}(a B+B a) \in V_{3}$ & $\vec{a} \cdot(\vec{b} \times \vec{c})$ \\
$B_{1} \cdot B_{2}=-\frac{1}{2}\left(B_{1} B_{2}+B_{2} B_{1}\right) \in V_{0}(\vec{a} \times \vec{b}) \cdot(\vec{c} \times \vec{d})$ \\
{$\left[B_{1}, B_{2}\right]=\frac{1}{2}\left(B_{1} B_{2}-B_{2} B_{1}\right) \in V_{2}(\vec{a} \times \vec{b}) \times(\vec{c} \times \vec{d})$} \\
$T_{1} \cdot T_{2}=-\frac{1}{2}\left(T_{1} T_{2}+T_{2} T_{1}\right) \in V_{0}$ & {$[\vec{a} \cdot(\vec{b} \times \vec{c})][\vec{f} \cdot(\vec{g} \times \vec{h})]$} \\
$B \cdot T=-\frac{1}{2}(B T+T B) \in V_{1}$ & $(\vec{a} \times \vec{b})[\vec{f} \cdot(\vec{g} \times \vec{h})]$ \\
$a \cdot T=\frac{1}{2}(a T+T a) \in V_{2}$ & $\vec{a}[\vec{f} \cdot(\vec{g} \times \vec{h})]$
\end{tabular}

Products between multivectors Other interior and exterior products between two multivectors $A_{p}$ and $B_{q}$ are defined for $p \leq q[4]$

$$
\begin{aligned}
A_{p} \cdot B_{q} & \equiv\left(v_{1} \wedge v_{2} \wedge \cdots \wedge v_{p-1}\right) \cdot\left(v_{p} \cdot B_{q}\right) \\
A_{p} \wedge B_{q} & \left.\equiv v_{1} \wedge\left(v_{2} \wedge \cdots \wedge v_{p}\right) \wedge B_{q}\right)
\end{aligned}
$$

with

$$
A_{p} \cdot B_{q}=(-1)^{p(q+1)} B_{q} \cdot A_{p}
$$

which defines $B_{q} \cdot A_{p}$ for $q \geq p$. The various products are given in Table 1 .

Associativity A major property of the exterior product is its associativity which is expressed as (with $v_{i} \in V_{1}$ ) [4].

$$
\left(v_{1} \wedge v_{2}\right) \wedge v_{3}=v_{1} \wedge\left(v_{2} \wedge v_{3}\right)
$$

Proof.

$$
\begin{aligned}
\left(v_{1} \wedge v_{2}\right) \wedge v_{3} & =v_{3} \wedge\left(v_{1} \wedge v_{2}\right) \\
& =\frac{1}{2}\left[-v_{3}\left(v_{1} \wedge v_{2}\right)-\left(v_{1} \wedge v_{2}\right) v_{3}\right] \\
& =\frac{1}{4}\left[v_{3}\left(v_{1} v_{2}-v_{2} v_{1}\right)+\left(v_{1} v_{2}-v_{2} v_{1}\right) v_{3}\right] \\
v_{1} \wedge\left(v_{2} \wedge v_{3}\right) & =\frac{1}{2}\left[-v_{1}\left(v_{2} \wedge v_{3}\right)-\left(v_{2} \wedge v_{3}\right) v_{1}\right] \\
& =\frac{1}{4}\left[v_{1}\left(v_{2} v_{3}-v_{3} v_{2}\right)+\left(v_{2} v_{3}-v_{3} v_{2}\right) v_{1}\right] .
\end{aligned}
$$


Since $v_{3} v_{1} v_{2}-v_{2} v_{1} v_{3}=-v_{1} v_{3} v_{2}+v_{2} v_{3} v_{1}$ because of

$$
\left(v_{3} v_{1}+v_{1} v_{3}\right) v_{2}=v_{2}\left(v_{3} v_{1}+v_{1} v_{3}\right),
$$

Eq. (37) is established.

\subsection{General formulas}

Among general formulas, one has with $(a, b, c, d) \in V_{1},\left(B, B_{i}\right) \in V_{2}$ and $F, G, H$ being any elements

$$
\begin{gathered}
(a \wedge b) \cdot B=a \cdot(b \cdot B)=-b \cdot(a \cdot B) \\
(a \wedge b) \cdot(c \wedge d)=(a \cdot c)(b \cdot d)-(a \cdot d)(b \cdot c) \\
{[F,[G, H]]-[G,[F, H]]=[[F, G], H]} \\
B_{2} \cdot\left(B_{1} \cdot a\right)-B_{1} \cdot\left(B_{2} \cdot a\right)=\left[B_{2}, B_{1}\right] \cdot a \\
a \cdot A_{p}=\left(a \wedge A_{p}^{*}\right)^{*} \\
a \wedge A_{p}=\left(a \cdot A_{p}^{*}\right)^{*} \\
B_{1} \wedge B_{2}^{*}=\left(B_{1} \cdot B_{2}\right)^{*} \\
B_{1} \cdot B_{2}=B_{1}^{*} \cdot B_{2}^{*}
\end{gathered}
$$

Proof. Eq. (44) results from the definition (34). Eq. (45) follows from

$$
(a \wedge b) \cdot(c \wedge d)=a \cdot[b \cdot(c \wedge d)]
$$

with

$$
\text { b. }(c \wedge d)=(b . d) c-(b . c) d
$$

hence,

$$
a .[b .(c \wedge d)]=(a . c)(b . d)-(b . c)(a . d) .
$$

Eq. (46) is simply the Jacobi identity which entails Eq. (47). Eq. (48) is established as follows (with $n=3$ )

$$
\begin{aligned}
a \wedge A_{p}^{*} & =a \wedge A_{n-p} \\
& =-\frac{1}{2}\left[a A_{n-p}+(-1)^{n-p} A_{n-p} a\right] \\
& =-\frac{1}{2}\left[a A_{n-p}-(-1)^{p} A_{n-p} a\right] \\
& =-\frac{I}{2}\left[a A_{p}-(-1)^{p} A_{p} a\right]=-\left(a \cdot A_{p}\right)^{*}
\end{aligned}
$$

hence, we obain since $\left(A^{*}\right)^{*}=-A$ the relation

$$
\left(a \wedge A_{p}^{*}\right)^{*}=\left(a \cdot A_{p}\right) .
$$


Eq. (49) follows from

$$
\begin{aligned}
a . A_{p}^{*} & =a \cdot A_{n-p} \\
& =\frac{1}{2}\left[a A_{n-p}-(-1)^{n-p} A_{n-p} a\right] \\
& =\frac{1}{2}\left[a A_{n-p}+(-1)^{p} A_{n-p} a\right] \\
& =\frac{I}{2}\left[a A_{p}+(-1)^{p} A_{p} a\right]
\end{aligned}
$$

thus we get

$$
\left(a . A_{p}^{*}\right)^{*}=\frac{-1}{2}\left[a A_{p}+(-1)^{p} A_{p} a\right]=a \wedge A_{p} .
$$

Eq. (50) results from

$$
\begin{aligned}
B_{1} \wedge B_{2}^{*} & =B_{2}^{*} \wedge B_{1} \\
& =\frac{-I}{2}\left(B_{2} B_{1}+B_{1} B_{2}\right)=\left(B_{1} \cdot B_{2}\right)^{*}
\end{aligned}
$$

and Eq. (51) from

$$
\begin{aligned}
\left(B_{1} \cdot B_{2}\right) & =\frac{-1}{2}\left(B_{1} B_{2}+B_{2} B_{1}\right) \\
& =\frac{1}{2}\left(I B_{1} I B_{2}+I B_{2} I B_{1}\right)=\left(B_{1}^{*} \cdot B_{2}^{*}\right) .
\end{aligned}
$$

\section{Multivector geometry}

\subsection{Analytic geometry}

The equation of a straight line parallel to the vector $u$ and going through the point $a$ is expressed by

$$
(x-a) \wedge u=0
$$

yielding the solution

$$
\begin{aligned}
x-a & =\lambda u \\
x & =\lambda u+a \quad(\lambda \in \mathbb{R}) .
\end{aligned}
$$

Similarly, the equation of a plane going through the point $a$ parallel to the plane $B=u \wedge v$ is expressed by

$$
(x-a) \wedge(u \wedge v)=0
$$

with the solution

$$
\begin{aligned}
x-a & =\lambda u+\mu v \\
x & =\lambda u+\mu v+a \quad(\lambda, \mu \in \mathbb{R})
\end{aligned}
$$




\subsection{Orthogonal projections}

Orthogonal projection of a vector on a vector The orthogonal projection of a vector $u=u_{\|}+u_{\perp}$ on a vector $a$ with $u_{\perp} \cdot a=0, u_{\|} \wedge a=0$ is obtained as follows. Since

$$
u a=u \cdot a-u \wedge a
$$

one has

$$
\begin{aligned}
u_{\|} a & =u_{\|} \cdot a=u \cdot a \\
u_{\perp} a & =-u_{\perp} \wedge a=-u \wedge a
\end{aligned}
$$

therefore

$$
\begin{aligned}
u_{\|} & =(u \cdot a) a^{-1} \\
u_{\perp} & =-(u \wedge a) a^{-1} .
\end{aligned}
$$

Orthogonal projection of a vector on a plane Similarly, to obtain the orthogonal projection of a vector $u=u_{\|}+u_{\perp}$ on a plane $B=a \wedge b$ (with $u_{\perp} \cdot B=0, u_{\|} \wedge B=0$ ) one writes

$$
u B=u \cdot B-u \wedge B
$$

hence, the solution is (with $B^{-1}=B_{c} / B B_{c}$ )

$$
\begin{aligned}
u_{\|} & =(u \cdot B) B^{-1} \\
u_{\perp} & =-(u \wedge B) B^{-1} .
\end{aligned}
$$

Orthogonal projection of a plane on a plane As another example, let us give the orthogonal projection of a plane $B_{1}=B_{1 \|}+B_{1 \perp}$ on the plane $B_{2}=a \wedge b$ with $B_{1 \perp} \cdot B_{2}=0$, and $\left[B_{1 \|}, B_{2}\right]=0$. Using the relation

$$
B_{1} B_{2}=-B_{1} \cdot B_{2}+\left[B_{1}, B_{2}\right]
$$

we obtain

$$
\begin{aligned}
B_{1 \|} & =-\left(B_{1} \cdot B_{2}\right) B_{2}^{-1} \\
B_{1 \perp} & =\left\{\left[B_{1}, B_{2}\right]\right\} B_{2}^{-1} .
\end{aligned}
$$

\section{Differential operators and integrals}

\subsection{Differential operators}

In Cartesian coordinates, the nabla operator $\nabla=I i \frac{\partial}{\partial x_{1}}+I j \frac{\partial}{\partial x_{2}}+I k \frac{\partial}{\partial x_{3}}$ acting on a scalar $f$, a vector $a\left(=a_{1} I i+a_{2} I j+a_{3} I k\right)$, a bivector $B\left(=B_{1} i+B_{2} j+B_{3} k\right)$ and a trivector $T(=\tau I)$ yields respectively

$$
\nabla f=I i \frac{\partial}{\partial x_{1}}+I j \frac{\partial}{\partial x_{2}}+I k \frac{\partial}{\partial x_{3}}=\operatorname{grad} f \in V_{1}
$$




$$
\begin{gathered}
\nabla \cdot a=\frac{\partial a_{1}}{\partial x_{1}}+\frac{\partial a_{2}}{\partial x_{2}}+\frac{\partial a_{3}}{\partial x_{3}}=\operatorname{diva} \in V_{0} \\
\nabla \wedge a=\left(\frac{\partial a_{3}}{\partial x_{2}}-\frac{\partial a_{2}}{\partial x_{3}}\right) i+\left(\frac{\partial a_{1}}{\partial x_{3}}-\frac{\partial a_{3}}{\partial x_{2}}\right) j+\left(\frac{\partial a_{2}}{\partial x_{1}}-\frac{\partial a_{1}}{\partial x_{2}}\right) k \\
=\operatorname{rot} a \in V_{2} \\
\nabla \cdot B=\left(\nabla \wedge B^{*}\right)^{*}=\left(\operatorname{rot} B^{*}\right)^{*} \in V_{1} \\
\nabla \wedge B=\left(\nabla \cdot B^{*}\right)^{*}=\left(\operatorname{div} B^{*}\right)^{*} \in V_{3} \\
\nabla \cdot T=\nabla T=-\left(\nabla T^{*}\right)^{*}=-\left(\operatorname{grad} T^{*}\right)^{*} \in V_{2} .
\end{gathered}
$$

Hence, the various operators can be expressed with the usual ones (grad, div, rot) and the duality. Among a few properties of the nabla operator, one has

$$
\begin{gathered}
\nabla^{2}=\triangle, \nabla \wedge(\nabla \wedge f)=0, \nabla \wedge(\nabla \wedge a)=0 \\
\triangle a=\nabla(\nabla a)=\nabla(\nabla \cdot a-\nabla \wedge a)=\nabla(\nabla \cdot a)-\nabla \cdot(\nabla \wedge a),
\end{gathered}
$$

where the last equation results from

$$
\nabla(\nabla \wedge a)=\nabla \cdot(\nabla \wedge a)-\nabla \wedge(\nabla \wedge a)=\nabla \cdot(\nabla \wedge a) .
$$

\subsection{Integrals and theorems}

The length, surface and volume integrals are respectively for a curve $x(u)$, surface $x(u, v)$ and a volume $x(u, v, w)$

$$
\begin{gathered}
L=\int d s=\int \sqrt{(d x)^{2}}=\int \sqrt{\left(\frac{d x}{d u}\right)^{2}} d u \\
S=\iint \sqrt{-\left(\frac{\partial x}{\partial u} \wedge \frac{\partial x}{\partial v}\right)^{2}} d u d v \\
V=\iiint \sqrt{-\left(\frac{\partial x}{\partial u} \wedge \frac{\partial x}{\partial v} \wedge \frac{\partial x}{\partial w}\right)^{2}} d u d v d w .
\end{gathered}
$$

The formulas exhibit immediately the transformation properties under a change of coordinates.

Stokes' theorem is expressed for a vector $a\left(\right.$ with $\left.d l=d x, d S=d l_{1} \wedge d l_{2}\right)$

$$
\oint a . d l=\int(\nabla \wedge a) \cdot d S
$$

the same formula can be used for a bivector $B$ by taking $a=B^{*}$

$$
\begin{aligned}
\oint B^{*} \cdot d l & =\int\left(\nabla \wedge B^{*}\right) \cdot d S=-\int(\nabla \cdot B)^{*} \cdot d S \\
& =\int \operatorname{rot} B^{*} \cdot d S .
\end{aligned}
$$


Ostrogradsky's theorem for a bivector $B$ yields (with $d \tau=d l_{1} \wedge d l_{2} \wedge d l_{3}$ )

$$
\begin{aligned}
\oint B \cdot d S & =\int(\nabla \wedge B) \cdot d \tau=\int\left(\operatorname{div} B^{*}\right)^{*} \cdot d \tau \\
& =\int\left(\operatorname{div} B^{*}\right) d V .
\end{aligned}
$$

For a vector $a$, one obtains with $B=-a^{*}$

$$
-\oint a^{*} \cdot d S=\int(\operatorname{diva}) d V
$$

which transforms, since $a^{*} . d S=-a . d S^{*}$, into

$$
\oint a . d S^{*}=\int(\operatorname{diva}) d V \text {. }
$$

\section{Orthogonal groups $O(3)$ and $S O(3)$}

Definition 2. The symmetric of $x$ with respect to a plane is obtained by drawing the perpendicular to the plane and by extending this perpendicular by an equal length.

Let $x$ be a vector, $x^{\prime}$ its symmetric to a plane and $a$ a unit vector perpendicular to the plane. From the geometry, $x^{\prime}-x$ is perpendicular to the plane and thus parallel to $a$; similarly, $x^{\prime}+x$ is parallel to the plane and thus perpendicular to $a$. Consequently, one has

$$
x^{\prime}=x+\lambda a, a \cdot\left(\frac{x^{\prime}+x}{2}\right)=0 ;
$$

hence, one obtains (with $a \cdot a=a^{2}=1$ )

$$
a \cdot\left(x+\frac{\lambda a}{2}\right)=0
$$

yielding $\lambda=-\frac{2(a \cdot x)}{a \cdot a}$ and

$$
\begin{aligned}
x^{\prime} & =x-\frac{2(a \cdot x) a}{a \cdot a}=x-\frac{(a x+x a) a}{a \cdot a} \\
& =-a x a .
\end{aligned}
$$

Definition 3. The orthogonal group $O(3)$ is the group of linear operators which leave invariant the quadratic form $x \cdot y=x_{1} y_{1}+x_{2} y_{2}+x_{3} y_{3}$

Theorem 2. Every rotation of $O(3)$ is the product of an even number $\leq 3$ of symmetries, any reflection is the product of an odd number $\leq 3$ of symmetries. 
The special orthogonal group $S O(3)$ is constituted by rotations i.e. of proper transformations $f(x)$ of determinant equal to 1 (i.e. $\alpha=f\left(e_{1}\right) \wedge f\left(e_{2}\right) \wedge f\left(e_{3}\right)=$ $I)$. A reflection is an improper transformation of determinant equal to -1 (i.e. $\alpha=-I)$. Combining two orthogonal symmetries, we obtain

$$
x^{\prime}=(b a) x(a b)=r x r_{c}
$$

with $r=b a, r_{c}=a_{c} b_{c}=a b, r r_{c}=1$. One can express $r$ as

$$
r=\left(\cos \frac{\theta}{2}+u \sin \frac{\theta}{2}\right)=e^{\frac{1}{2} u \theta}
$$

with $u=u_{1} i+u_{2} j+u_{3} k\left(u^{2}=-1\right)$. Eq. (110) represents a conical rotation of the vector $x$ by an angle $\theta$ around the unit vector $u^{*}=I u$. One verifies that the rotation conserves the norm $x^{\prime 2}=x^{2}$. The same equation holds for any element $A$ of the algebra $A^{\prime}=r A r_{c}$ since the product of two vectors $x, y$ transforms as

$$
x^{\prime} y^{\prime}=\left(r x r_{c}\right)\left(r y r_{c}\right)=r(x y) r_{c}
$$

and similarly for the product of three vectors as well as a linear combination of such products. The above formulas allow to easily express the classical moving frames such as the Frenet and Darboux frames within the Grassmannian scheme.

\section{Curves}

\subsection{Generalities}

Consider a $3 D$ curve $x(t)\left(=x_{1}(t) e_{1}+x_{2}(t) e_{2}+x_{3}(t) e_{3}\right)$ where $e_{i}$ is the canonical orthonormal basis $\left(e_{1}=I i, e_{2}=I j, e_{3}=I k\right)$. Taking the length of the curve $s$ as parameter we have $x=f(s)$ with $d s=\sqrt{(d x)^{2}}$. The tangent unit vector at a point $M(x)$ is

$$
T=\frac{d x}{d s}, T^{2}=\left(\frac{d x}{d s}\right)^{2}=1 .
$$

The equation of the tangent at a point $M(x)$ is given by

$$
(X-x) \wedge \frac{d x}{d s}=0
$$

where $X$ is a generic point of the tangent. The equations of the plane perpendicular to the curve and of the osculating plane at the point $M$ read respectively

$$
\begin{gathered}
(X-x) \cdot \frac{d x}{d s}=0 \\
(X-x) \wedge \frac{d x}{d s} \wedge \frac{d^{2} x}{d s^{2}}=0
\end{gathered}
$$

where $X$ is a generic point of the plane [25]. 


\subsection{Frenet frame}

The Frenet frame $\left(v_{i}\right)$ attached to the curve $x(t)$ is given by

$$
v_{i}=r e_{i} r_{c}
$$

where $r=e^{\frac{1}{2} u \theta}$ expresses the rotation of angle $\theta$ around the unit vector $u^{*}$ $\left(r r_{c}=1, u^{2}=-1\right)$ and $e_{i}$ is the canonical orthonormal basis. After differentiation, one obtains (using the relation $d r_{c} r=-r_{c} d r$ resulting from the differentiation of $r r_{c}=1$ )

$$
\begin{aligned}
d v_{i} & =r\left(r_{c} d r e_{i}+e_{i} d r_{c} r\right) r_{c} \\
& =r\left(d \iota_{F} \cdot e_{i}\right) r_{c} \\
& =r\left(D e_{i}\right) r_{c}
\end{aligned}
$$

with

$$
\begin{aligned}
d \iota_{F} & =2 r_{c} d r=2 e^{-\frac{1}{2} u \theta} e^{\frac{1}{2} u \theta}\left(\frac{d \theta}{2} u+\frac{\theta}{2} d u\right) \\
& =(d \theta u+\theta d u) \\
& =(d a) i+(d b) j+(d c) k) \in V_{2}
\end{aligned}
$$

and

$$
D e_{i}=d \iota_{F} \cdot e_{i}=\frac{1}{2}\left(d \iota_{F} e_{i}-e_{i} d \iota_{F}\right) .
$$

We shall call $d \iota_{F}=2 r_{c} d r$ the affine connection bivector. Explicitly, one has

$$
\begin{aligned}
& D e_{1}=(d c) e_{2}-(d b) e_{3} \\
& D e_{2}=-(d c) e_{1}+(d a) e_{3} \\
& D e_{3}=(d b) e_{1}-(d a) e_{2} .
\end{aligned}
$$

The Frenet frame is defined by the affine connection bivector

$$
d \iota_{F}=2 r_{c} d r=(\tau d s) i+(\rho d s) k
$$

where $\rho=1 / R$ is the curvature and $\tau=1 / T$ the torsion. This gives the Frenet equations

$$
\begin{aligned}
& D e_{1}=(\rho d s) e_{2} \\
& D e_{2}=-(\rho d s) e_{1}+(\tau d s) e_{3} \\
& D e_{3}=(-\tau d s) e_{2} .
\end{aligned}
$$

\subsection{Curvature and torsion}

To obtain the curvature and torsion we define $\alpha$ and $\beta$

$$
\alpha=\frac{d x}{d s} \wedge \frac{d^{2} x}{d s^{2}}=\rho v_{1} \wedge v_{2}
$$




$$
\beta=\frac{d x}{d s} \wedge \frac{d^{2} x}{d s^{2}} \wedge \frac{d^{3} x}{d s^{3}}=I \rho^{2} \tau
$$

and using the Lagrange equation

$$
\left(v_{1} \wedge v_{2}\right)^{2}=\left(v_{1} \cdot v_{2}\right)^{2}-\left(v_{1}\right)^{2}\left(v_{2}\right)^{2}=-1
$$

we obtain the invariants

$$
\begin{gathered}
\rho=\sqrt{-\alpha^{2}}=\sqrt{-\left(\frac{d x}{d s} \wedge \frac{d^{2} x}{d s^{2}}\right)^{2}} \\
\tau=\frac{I \beta}{\alpha^{2}}=\frac{I\left(\frac{d x}{d s} \wedge \frac{d^{2} x}{d s^{2}} \wedge \frac{d^{3} x}{d s^{3}}\right)}{\left(\frac{d x}{d s} \wedge \frac{d^{2} x}{d s^{2}}\right)^{2}} .
\end{gathered}
$$

Under a change of parameter $t$, one has using $\frac{d x}{d t}=\frac{d x}{d s} \frac{d s}{d t}$

$$
\frac{d x}{d t} \wedge \frac{d^{2} x}{d t^{2}}=\left(\frac{d s}{d t}\right)^{3} \alpha
$$

and thus one obtains the curvature

$$
\rho=\sqrt{-\left(\frac{d x}{d t}\right)^{-6}\left(\frac{d x}{d t} \wedge \frac{d^{2} x}{d t^{2}}\right)^{2}} .
$$

For the torsion, proceeding similarly, we get under a change of parameter

$$
\frac{d x}{d t} \wedge \frac{d^{2} x}{d t^{2}} \wedge \frac{d^{3} x}{d t^{3}}=\left(\frac{d s}{d t}\right)^{6} \beta
$$

and thus

$$
\tau=\frac{I\left(\frac{d x}{d t} \wedge \frac{d^{2} x}{d t^{2}} \wedge \frac{d^{3} x}{d t^{3}}\right)}{\left(\frac{d x}{d t} \wedge \frac{d^{2} x}{d t^{2}}\right)^{2}} \in V_{0} .
$$

\subsection{Example}

As example, consider the curve $x(t)=(2 \cos t) I i+(2 \sin t) I j+(t) I k$. The line element is $d s=\sqrt{d x^{2}}=\sqrt{5} d t$; writing $x^{\prime}=\frac{d x}{d t}$, etc., we have

$$
\begin{aligned}
x^{\prime} \wedge x^{\prime \prime} & =(2 \sin t) i-(2 \cos t) j+4 k, \\
\left(x^{\prime} \wedge x^{\prime \prime}\right)^{2} & =-20 \\
x^{\prime} \wedge x^{\prime \prime} \wedge x^{\prime \prime \prime} & =4 I .
\end{aligned}
$$

The curvature and torsion are respectively

$$
\begin{aligned}
& \rho=\sqrt{-\left(x^{\prime}\right)^{-6}\left(x^{\prime} \wedge x^{\prime \prime}\right)^{2}}=\frac{2}{5} \\
& \tau=\frac{I\left(x^{\prime} \wedge x^{\prime \prime} \wedge x^{\prime \prime \prime}\right)}{\left(x^{\prime} \wedge x^{\prime \prime}\right)^{2}}=\frac{1}{5} .
\end{aligned}
$$


The equation of the osculating plane is (with $X=\left(X_{1}\right) I i+\left(X_{2}\right) I j+\left(X_{3}\right) I k$ being a generic point of the plane )

$$
(X-x) \wedge \frac{d x}{d s} \wedge \frac{d^{2} x}{d s^{2}}=\frac{I}{\sqrt{5}}\left(-2 t+2 X_{3}-X_{2} \cos t+X_{1} \sin t\right)=0 .
$$

The Frenet basis $v_{i}$ is

$$
\begin{aligned}
& v_{1}=\frac{1}{\sqrt{5}}[(-2 \sin t) I i+(2 \cos t) I j+I k] \\
& v_{2}=(-\cos t) I i-(\sin t) I j \\
& v_{3}=\left(v_{1} \wedge v_{2}\right)^{*}=\frac{1}{\sqrt{5}}[(\sin t) I i-(\cos t) I j+2 I k] .
\end{aligned}
$$

The basis $v_{i}$ is obtained via the following rotations. First, the frame is brought into its initial position (at $t=0$ ) via the rotation $f_{0}=f_{1} f_{2}$ with $\tan \theta=\frac{1}{2}$ and

$$
\begin{aligned}
& f_{1}=e^{k \frac{\pi}{2}}=\frac{1}{\sqrt{2}}(1+k) \\
& f_{2}=e^{-j \frac{\theta}{2}}=\left(\sqrt{\frac{1}{\sqrt{2}}+\frac{1}{\sqrt{5}}}-j \sqrt{\frac{1}{\sqrt{2}}-\frac{1}{\sqrt{5}}}\right)
\end{aligned}
$$

yielding

$$
f_{0}=\sqrt{\left(\frac{1}{4}+\frac{1}{2 \sqrt{5}}\right)}+i \sqrt{\frac{1}{4}-\frac{1}{2 \sqrt{5}}}-j \sqrt{\frac{1}{4}-\frac{1}{2 \sqrt{5}}}+k \sqrt{\frac{1}{4}+\frac{1}{2 \sqrt{5}}} .
$$

Next, follows the rotation due to the affine connection bivector

$$
f_{3}=\cos \frac{t}{2}+\left(\frac{i}{\sqrt{5}}+\frac{2 k}{\sqrt{5}}\right) \sin \frac{t}{2} .
$$

The end result is $r=f_{1} f_{2} f_{3}$ and explicitly

$$
\begin{aligned}
r= & \left(A \cos \frac{t}{2}-C \sin \frac{t}{2}\right)+i\left(B \cos \frac{t}{2}+D \sin \frac{t}{2}\right) \\
& +j\left(-B \cos \frac{t}{2}+D \sin \frac{t}{2}\right)+k\left(A \cos \frac{t}{2}+C \sin \frac{t}{2}\right)
\end{aligned}
$$

with

$$
\begin{aligned}
& A=\frac{1}{10} \sqrt{5(5+2 \sqrt{5})}, B=\frac{1}{10} \sqrt{5(5-2 \sqrt{5})} \\
& C=\frac{1}{10}(\sqrt{5-2 \sqrt{5}}+2 \sqrt{5+2 \sqrt{5})}, \\
& D=\frac{1}{10}(-2 \sqrt{5-2 \sqrt{5}}+\sqrt{5+2 \sqrt{5})} .
\end{aligned}
$$

Finally, one verifies that $v_{i}=r e_{i} r_{c}$. 


\section{Surfaces}

\subsection{Generalities}

Consider in a $3 D$ Euclidean space a surface $x=f(u, v)$. The tangent plane is given by $f_{u} \wedge f_{v}\left(f_{u}=\frac{\partial f}{\partial u}, f_{v}=\frac{\partial f}{\partial v}\right)$ and the unit normal by

$$
h=\frac{\left(f_{u} \wedge f_{v}\right)^{*}}{\sqrt{-\left(f_{u} \wedge f_{v}\right)^{2}}}
$$

with $\left(f_{u}, f_{v}, h\right)$ being a direct trieder $\left(f_{u} \wedge f_{v} \wedge h=\lambda I, \lambda>0\right)$. Take an orthonormal moving frame of basis vectors $v_{i}$ and a vector $a$ (of components $A$ with respect to the moving frame) attached to the surface. This frame is obtained by rotating the canonical frame $e_{i}$; hence, one has

$$
v_{i}=r e_{i} r_{c}, a=r A r_{c}
$$

Differentiating these relations, one obtains $d v_{i}=r\left(D e_{i}\right) r_{c}, d a=r(D A) r_{c}$ with

$$
D e_{i}=d \iota . e_{i}, D A=d A+d \iota . A
$$

where $D A$ is the covariant differential ( $d A$ being a differentiation with respect to the components only) and $d \iota=2 r_{c} d r$.

\subsection{Darboux frame}

The Darboux frame $\left(v_{i D}\right)$ is obtained for a curve on the surface from the Frenet frame by a rotation of an algebraic angle $\alpha=\angle\left(v_{3 F}, h\right)$ around $v_{1 F}$. Hence

$$
v_{i D}=\left(r_{D}\right) e_{i}\left(r_{D}\right)_{c}
$$

with $r_{D}=r_{1} r$ and

$$
r_{1}=\cos \frac{\alpha}{2}-\left(v_{1 F}\right)^{*} \sin \frac{\alpha}{2}=r p r_{c}
$$

where $p=\cos \frac{\alpha}{2}+i \sin \frac{\alpha}{2}$; hence $r_{D}=r_{1} r=r p$. Consequently, the rotation bivector $d \iota_{D}=2\left(r_{D}\right)_{c} d r_{D}$ can be expressed as follows

$$
\begin{aligned}
d \iota_{D} & =2(r p)_{c} d(r p)=2 p_{c} r_{c}(d r p+r d p) \\
& =p_{c}\left(d \iota_{F}\right) p+2 p_{c} d p \\
& =\left(\tau_{r} d s\right) i-\left(\rho_{n} d s\right) j+\left(\rho_{g} d s\right) k
\end{aligned}
$$

where $\rho_{g}=\rho \cos \alpha$ is the geodesic curvature, $\rho_{g}=-\rho \sin \alpha$ the normal curvature and $\tau_{r}=\frac{d \alpha}{d s}+\tau$ the relative torsion. The Darboux equations thus become

$$
d v_{i D}=r_{D}\left(d \iota_{D} \cdot e_{i}\right)\left(r_{D}\right)_{c}
$$


and explicitly

$$
\begin{aligned}
& d v_{1 D}=\left(\rho_{g} v_{2 D}+\rho_{n} v_{3 D}\right) d s \\
& d v_{2 D}=\left(-\rho_{g} v_{1 D}+\tau_{r} v_{3 D}\right) d s \\
& d v_{3 D}=\left(-\rho_{n} v_{1 D}-\tau_{r} v_{2 D}\right) d s .
\end{aligned}
$$

If the Darboux frame is rotated in the tangent plane by an angle $\theta(s)$ around the unit normal $h$ one obtains, applying the same reasoning as above, for the rotation $r=r_{D} f$ with $f=\cos \frac{\theta}{2}+k \sin \frac{\theta}{2}$. The new vectors $v_{i}$ of the frame become $v_{i}=r e_{i} r_{c}$ and the affine connection bivector transforms into

$$
\begin{aligned}
d \iota & =2 r_{c} d r \\
& =2 f_{c}\left(r_{D}\right)_{c}\left[\left(d r_{D}\right) f+r_{D} d f\right] \\
& =f_{c}\left(d \iota_{D}\right) f+2 f_{c} d f .
\end{aligned}
$$

Applying this formula, one obtains

$$
\begin{aligned}
d \iota= & d s\left(\tau_{r} \cos \theta-\rho_{n} \sin \theta\right) i+d s\left(-\rho_{n} \cos \theta-\tau_{r} \sin \theta\right) j \\
& +\left(\rho_{g} d s+d \theta\right) k .
\end{aligned}
$$

\subsection{Integrability conditions}

The $3 D$ Euclidean space being without torsion and curvature, this entails that $d M$ and $d v_{i}$ are integrable.

Integrability of $\boldsymbol{d} \boldsymbol{M}$ Consider a point on the surface, one has

$$
d M=r(D M) r_{c}=f_{u} d u+f_{v} d v
$$

where $D M$ are the components expressed in the moving frame (with the moving vectors $\left.v_{i}=r\left(e_{i}\right) r_{c}\right)$. One has

$$
\begin{aligned}
D M & =\omega_{1} e_{1}+\omega_{2} e_{2} \\
& =\left(A_{1} d u+B_{1} d v\right) e_{1}+\left(A_{2} d u+B_{2} d v\right) e_{2}
\end{aligned}
$$

with

$$
\begin{aligned}
& A_{1}=\left(r_{c} f_{u} r\right) \cdot e_{1}=f_{u} \cdot v_{1}, B_{1}=\left(r_{c} f_{v} r\right) \cdot e_{1}=f_{v} \cdot v_{1} \\
& A_{2}=\left(r_{c} f_{u} r\right) \cdot e_{2}=f_{u} \cdot v_{2}, B_{2}=\left(r_{c} f_{v} r\right) \cdot e_{2}=f_{v} \cdot v_{2} .
\end{aligned}
$$

The affine connection bivector $d \iota=2 r_{c} d r$ can be written [15, II, p. 410] as $d \iota=\iota_{1} d u+\iota_{2} d v$ (with $\iota_{1}=a_{1} i+b_{1} j+c_{1} k, \iota_{2}=a_{2} i+b_{2} j+c_{2} k$ ). The integrability condition of $d M$ is expressed by the condition

$$
\Delta(D M)-D(\Delta M)=0
$$


with

$$
\begin{aligned}
& \Delta(D M)=\delta(D M)+\delta \iota . D M \\
& D(\Delta M)=d(\Delta M)+d \iota . \Delta M .
\end{aligned}
$$

This leads to the relation

$$
\delta(D M)-d(\Delta M)=d \iota . \Delta M-\delta \iota . D M .
$$

Explicitly, it reads

$$
\begin{aligned}
& \frac{\partial A_{1}}{\partial v}-\frac{\partial B_{1}}{\partial u}=-B_{2} c_{1}+A_{2} c_{2} \\
& \frac{\partial A_{2}}{\partial v}-\frac{\partial B_{2}}{\partial u}=B_{1} c_{1}-A_{1} c_{2} \\
& A_{1} b_{2}+B_{2} a_{1}=A_{2} a_{2}+B_{1} b_{1} .
\end{aligned}
$$

The linear Eqs. $(185,186)$ determine $c_{1}, c_{2}$; the result is

$$
\begin{aligned}
& c_{1}=\frac{-1}{A_{1} B_{2}-A_{2} B_{1}}\left[A_{1}\left(\frac{\partial A_{1}}{\partial v}-\frac{\partial B_{1}}{\partial u}\right)+A_{2}\left(\frac{\partial A_{2}}{\partial v}-\frac{\partial B_{2}}{\partial u}\right)\right] \\
& c_{2}=\frac{-1}{A_{1} B_{2}-A_{2} B_{1}}\left[B_{2}\left(\frac{\partial A_{2}}{\partial v}-\frac{\partial B_{2}}{\partial u}\right)+B_{1}\left(\frac{\partial A_{1}}{\partial v}-\frac{\partial B_{1}}{\partial u}\right)\right]
\end{aligned}
$$

Integrability of $\boldsymbol{d} \boldsymbol{v}_{\boldsymbol{i}}$ The integrability conditions of $d v_{i}$ are obtained similarly, with $d v_{i}=r D e_{i} r_{c}$. The condition $\delta d v_{i}-d \delta v_{i}=0$ leads to the relation

$$
\Delta\left(D e_{i}\right)-D\left(\Delta e_{i}\right)=0
$$

with $D e_{i}=d \iota . e_{i}, \Delta e_{i}=\delta \iota . e_{i}$ and

$$
\begin{aligned}
& \Delta\left(D e_{i}\right)=\delta\left(d \iota . e_{i}\right)+\delta \iota .\left(d \iota . e_{i}\right) \\
& D\left(\Delta e_{i}\right)=d\left(\delta \iota . e_{i}\right)+d \iota .\left(\delta \iota . e_{i}\right) .
\end{aligned}
$$

Applying Eq. (47), one has

$$
\delta \iota .\left(d \iota . e_{i}\right)-d \iota .\left(\delta \iota \cdot e_{i}\right)=-[d \iota, \delta \iota] \cdot e_{i}
$$

hence, the integrability condition of $d v_{i}$ can be expressed as

$$
(\delta d \iota-d \delta \iota) \cdot e_{i}=[d \iota, \delta \iota] \cdot e_{i}
$$

and thus $(\delta d \iota-d \delta \iota)=[d \iota, \delta \iota]$ or

$$
\frac{\partial \iota_{1}}{\partial v}-\frac{\partial \iota_{2}}{\partial u}=\left[\iota_{1}, \iota_{2}\right] .
$$

Explicitly, these equations read [15, II, p. 412]

$$
\begin{aligned}
& \frac{\partial a_{1}}{\partial v}-\frac{\partial a_{2}}{\partial u}=b_{1} c_{2}-b_{2} c_{1} \\
& \frac{\partial b_{1}}{\partial v}-\frac{\partial b_{2}}{\partial u}=c_{1} a_{2}-c_{2} a_{1} \\
& \frac{\partial c_{1}}{\partial v}-\frac{\partial c_{2}}{\partial u}=a_{1} b_{2}-a_{2} b_{1} .
\end{aligned}
$$




\subsection{Curvature lines and curvature: first method}

Consider a moving frame $v_{i}=r e_{i} r_{c}$ with $D e_{i}=d \iota . e_{i}\left(d \iota=2 r_{c} d r\right)$ and $D M=$ $\omega_{1} e_{1}+\omega_{2} e_{2}$ expressed in the moving frame. The fundamental form $\Pi$ can be expressed as

$$
\begin{aligned}
\Pi & =-D e_{3} \cdot D M=-\left(d \iota \cdot e_{3}\right) \cdot D M \\
& =D M \cdot\left(e_{3} \cdot d \iota\right)=\left(D M \wedge e_{3}\right) \cdot d \iota .
\end{aligned}
$$

The affine connection bivector $d \iota=d a i+d b j+d c k$ can be developed on $\omega_{1}(=$ $\left.A_{1} d u+B_{1} d v\right), \omega_{2}\left(=A_{2} d u+B_{2} d v\right)$ as follows [26, p. 209]

$$
\begin{aligned}
d a & =L_{21} \omega_{1}+L_{22} \omega_{2}=a_{1} d u+a_{2} d v \\
d b & =-L_{11} \omega_{1}-L_{12} \omega_{2}=b_{1} d u+b_{2} d v .
\end{aligned}
$$

Identifying the coefficients of $d u, d v$, and solving the linear system, one obtains

$$
\begin{aligned}
& L_{11}=\frac{b_{2} A_{2}-b_{1} B_{2}}{A_{1} B_{2}-A_{2} B_{1}}, L_{22}=\frac{a_{2} A_{1}-a_{1} B_{1}}{A_{1} B_{2}-A_{2} B_{1}} \\
& L_{12}=\frac{b_{1} B_{1}-b_{2} A_{1}}{A_{1} B_{2}-A_{2} B_{1}}, L_{21}=\frac{a_{1} B_{2}-a_{2} A_{2}}{A_{1} B_{2}-A_{2} B_{1}} .
\end{aligned}
$$

Due to the integrability condition Eq. (187), we have $L_{12}=L_{21}$ and thus the fondamental form $\Pi$ becomes

$$
\Pi=L_{11} \omega_{1}^{2}+L_{22} \omega_{2}^{2}+2 L_{12} \omega_{1} \omega_{2}
$$

If we rotate the frame by an angle $\Phi$ around $v_{3}$ we have $v_{i}^{\prime}=f v_{i} f_{c}$ with

$$
\begin{aligned}
f & =\cos (\Phi / 2)-v_{3}^{*} \sin (\Phi / 2) \\
& =r\left[\cos (\Phi / 2)-e_{3}^{*} \sin (\Phi / 2)\right] r_{c} \\
& =r[\cos (\Phi / 2)+k \sin (\Phi / 2)] r_{c} .
\end{aligned}
$$

The total rotation is $R=f r=r p$ with $p=\cos (\Phi / 2)+k \sin (\Phi / 2)$ and the affine connection bivector transforms into

$$
\begin{aligned}
d \iota^{\prime} & =2 R_{c} d R=2 p_{c} r_{c}(d r p)=p_{c}(d \iota) p \\
& =d a^{\prime} i+d b^{\prime} j+d c^{\prime} k
\end{aligned}
$$

with

$$
\begin{gathered}
d a^{\prime}=\cos (\Phi) d a+\sin (\Phi) d b=L_{12}^{\prime} \omega_{1}^{\prime}+L_{22}^{\prime} \omega_{2}^{\prime} \\
d b^{\prime}=-\sin (\Phi) d a+\cos (\Phi) d b=-L_{11}^{\prime} \omega_{1}^{\prime}-L_{12}^{\prime} \omega_{2}^{\prime} \\
d c^{\prime}=d c .
\end{gathered}
$$

The vector $D M^{\prime}=\omega_{1}^{\prime} e_{1}+\omega_{2}^{\prime} e_{2}$ transforms in the same way i.e.,

$$
\omega_{1}^{\prime}=\cos (\Phi) \omega_{1}+\sin (\Phi) \omega_{2}
$$




$$
\omega_{2}^{\prime}=-\sin (\Phi) \omega_{1}+\cos (\Phi) \omega_{2} .
$$

The coefficients $L_{i j}^{\prime}$ are obtained by expressing $(d a, d b)$ of Eqs. $(211,212)$ in terms of $\left(L_{i j}, \omega_{i}\right)$ via the Eqs. $(201,202)$ and then by writing the $\omega_{i}$ in terms of $\omega_{i}^{\prime}$; one finds

$$
\begin{gathered}
L_{11}^{\prime}=L_{11} \cos ^{2}(\Phi)+L_{22} \sin ^{2}(\Phi)+L_{12} \sin (2 \Phi) \\
L_{22}^{\prime}=L_{11} \sin ^{2}(\Phi)+L_{22} \cos ^{2}(\Phi)-L_{12} \sin (2 \Phi) \\
L_{12}^{\prime}=L_{12} \cos (2 \Phi)+\frac{1}{2} \sin (2 \Phi)\left(L_{22}-L_{11}\right) .
\end{gathered}
$$

The curvature lines are the lines for which the fundamental form $\Pi$ becomes diagonal, i.e., when $L_{12}^{\prime}=0$, or

$$
\tan 2 \Phi=\frac{2 L_{12}}{\left(L_{11}-L_{22}\right)} .
$$

Along these lines, the curvature is defined by $D e_{3}=-K(D M)$ with

$$
\begin{aligned}
D e_{3} & =d b^{\prime} e_{1}-d a^{\prime} e_{2} \\
d a^{\prime} & =L_{22}^{\prime} \omega_{2}^{\prime}, d b=-L_{11}^{\prime} \omega_{1}^{\prime}
\end{aligned}
$$

and $D M=\omega_{1}^{\prime} e_{1}$ or $D M=\omega_{2}^{\prime} e_{2}$; hence, the curvatures are given by $K_{1}=$ $L_{11}^{\prime}, K_{2}=L_{22}^{\prime}$ i.e., as a function of the angle $\Phi$. To obtain the standard formulas, we write

$$
\cos 2 \Phi=\frac{L_{11}-L_{22}}{\left[\left(L_{11}-L_{22}\right)^{2}+4 L_{12}^{2}\right]^{1 / 2}}, \sin 2 \Phi=\frac{2 L_{12}}{\left[\left(L_{11}-L_{22}\right)^{2}+4 L_{12}^{2}\right]^{1 / 2}}
$$

and use $\cos ^{2} \Phi=\frac{1}{2}(1+\cos 2 \Phi), \sin ^{2} \Phi=\frac{1}{2}(1-\cos 2 \Phi)$; after rearrangement, we get

$$
\begin{aligned}
& K_{1}=L_{11}^{\prime}=\frac{1}{2}\left(L_{11}+L_{22}-\sqrt{\left(L_{11}-L_{22}\right)^{2}+4 L_{12}^{2}}\right) \\
& K_{2}=L_{12}^{\prime}=\frac{1}{2}\left(L_{11}+L_{22}+\sqrt{\left(L_{11}-L_{22}\right)^{2}+4 L_{12}^{2}}\right) .
\end{aligned}
$$

Hence, the Gaussian curvature $K$ is given by

$$
\begin{aligned}
K & =K_{1} K_{2}=L_{11} L_{22}-L_{12}^{2} \\
& =\frac{a_{1} b_{2}-a_{2} b_{1}}{A_{1} B_{2}-A_{2} B_{1}}=\frac{\left(\frac{\partial c_{1}}{\partial v}-\frac{\partial c_{2}}{\partial u}\right)}{A_{1} B_{2}-A_{2} B_{1}}
\end{aligned}
$$

where we have made use of the integrability condition Eq. (198). Replacing $c_{1}, c_{2}$ by their expressions of Eqs. $(188,189)$, we finally get for the Gaussian curvature

$$
K=\frac{1}{\left(A_{1} B_{2}-A_{2} B_{1}\right)}\left\{\begin{array}{c}
\frac{\partial}{\partial u}\left[\frac{B_{2}\left(\frac{\partial A_{2}}{\partial v}-\frac{\partial B_{2}}{\partial u}\right)+B_{1}\left(\frac{\partial A_{1}}{\partial v}-\frac{\partial B_{1}}{\partial u}\right)}{\left(A_{1} B_{2}-A_{2} B_{1}\right)}\right] \\
-\frac{\partial}{\partial v}\left[\frac{A_{1}\left(\frac{\partial A_{1}}{\partial v}-\frac{\partial B_{1}}{\partial u}\right)+A_{2}\left(\frac{\partial A_{2}}{\partial v}-\frac{\partial B_{2}}{\partial u}\right)}{\left(A_{1} B_{2}-A_{2} B_{1}\right)}\right]
\end{array}\right\}
$$


The Gaussian curvature thus depends only on the metric $d s=\sqrt{(d M)^{2}}$, as stated by Gauss' theorem. The mean curvature $H$ is given by

$$
\begin{aligned}
H & =\frac{1}{2}\left(K_{1}+K_{2}\right)=\frac{1}{2}\left(L_{11}+L_{22}\right) \\
& =\frac{-b_{1} B_{2}+b_{2} A_{2}+a_{2} A_{1}-a_{1} B_{1}}{2\left(A_{1} B_{2}-A_{2} B_{1}\right)} .
\end{aligned}
$$

\subsection{Gaussian and mean curvature: second method}

The Gaussian and mean curvatures can also be derived as follows [25]. The curvature $K$ is defined by the relation

$$
d v_{3}=-K d M
$$

Developing that equation we have

$$
\frac{\partial v_{3}}{\partial u} d u+\frac{\partial v_{3}}{\partial v} d v=-K\left(x_{u} d u+x_{v} d v\right)
$$

or

$$
\left(K x_{u}+\frac{\partial v_{3}}{\partial u}\right) d u+\left(K x_{v}+\frac{\partial v_{3}}{\partial v}\right) d v=0
$$

The two vectors in parentheses are parallel and thus

$$
\left(K x_{u}+\frac{\partial v_{3}}{\partial u}\right) \wedge\left(K x_{v}+\frac{\partial v_{3}}{\partial v}\right)=0
$$

which gives the equation

$$
K^{2}\left(x_{u} \wedge x_{v}\right)+K\left(\frac{\partial v_{3}}{\partial u} \wedge x_{v}+x_{u} \wedge \frac{\partial v_{3}}{\partial v}\right)+\frac{\partial v_{3}}{\partial u} \wedge \frac{\partial v_{3}}{\partial v}=0 .
$$

Multiplying with the exterior product on the left by $n=\left(x_{u} \wedge x_{v}\right)^{*}$ and using Eq. (50)

$$
\left(x_{u} \wedge x_{v}\right)^{*} \wedge\left(x_{u} \wedge x_{v}\right)=-\left[\left(x_{u} \wedge x_{v}\right)^{2}\right]^{*}=I\left(n^{2}\right)
$$

we obtain the formula $[25]$

$$
K^{2} I\left(n^{2}\right)+K\left[n \wedge \frac{\partial v_{3}}{\partial u} \wedge x_{v}+n \wedge x_{u} \wedge \frac{\partial v_{3}}{\partial v}\right]+n \wedge \frac{\partial v_{3}}{\partial u} \wedge \frac{\partial v_{3}}{\partial v}=0 .
$$

Calling $K_{1}, K_{2}$ the two roots of the equation, one has for the Gaussian curvature

$$
K=K_{1} K_{2}=\frac{-I}{n^{2}}\left(n \wedge \frac{\partial v_{3}}{\partial u} \wedge \frac{\partial v_{3}}{\partial v}\right) .
$$

The mean curvature is given by

$$
H=\frac{1}{2}\left(K_{1}+K_{2}\right)=\frac{I}{2 n^{2}}\left(n \wedge x_{u} \wedge \frac{\partial v_{3}}{\partial v}+n \wedge \frac{\partial v_{3}}{\partial u} \wedge x_{v}\right) .
$$




\subsection{Curves on surfaces: asymptotic, curvature and geodesic lines}

Consider on a surface, at a point $O M$, an orthonormal frame $v_{1}, v_{2}, v_{3}$ (with $v_{3}$ being the unit normal). One has (with $\omega_{1}=\cos \Phi d s, \omega_{2}=\sin \Phi d s$ ) [15, II, p. $413]$

$$
\begin{gathered}
d(O M)=\left(v_{1} \cos \Phi+v_{2} \sin \Phi\right) d s \\
=\omega_{1} v_{1}+\omega_{2} v_{2} \\
d^{2}(O M)=\frac{d^{2}(O M)}{d s^{2}} d s=\left(v_{2} d c-v_{3} d b\right) \cos \Phi \\
+\left(-v_{1} d c+v_{3} d a\right) \sin \Phi+\left(v_{2} \cos \Phi-v_{1} \sin \Phi\right) d \Phi \\
=-v_{1} \sin \Phi(d c+d \Phi)+v_{2} \cos \Phi(d c+d \Phi) \\
+v_{3}(\sin \Phi d a-\cos \Phi d b) .
\end{gathered}
$$

Asymptotic lines The normal curvature is defined by

$$
\begin{aligned}
\rho_{n}(s) & =\frac{d^{2}(O M)}{d s^{2}} \cdot v_{3} \\
& =\left(\sin \Phi \frac{d a}{d s}-\cos \Phi \frac{d b}{d s}\right) .
\end{aligned}
$$

Developing $d a, d b$ on $\omega_{1}, \omega_{2}$ via Eqs. $(201,202)$, one obtains after rearrangement

$$
\rho_{n}(s)=L_{22} \sin ^{2} \Phi+L_{11} \cos ^{2} \Phi+2 \sin \Phi \cos \Phi L_{12} .
$$

The asymptotic line is defined by $\rho_{n}(s)=0$, leading to

$$
\tan \Phi=\frac{-L_{12} \pm \sqrt{L_{12}^{2}-L_{11} L_{22}}}{L_{22}}
$$

under the assumption that $L_{12}^{2}-L_{11} L_{22} \geq 0$.

Curvature lines The relative torsion $\tau_{r}(s)$ is expressed by

$$
\begin{aligned}
I \tau_{r}(s) & =\frac{d(O M)}{d s} \wedge v_{3} \wedge \frac{d v_{3}}{d s} \\
& =\left(v_{1} \cos \Phi+v_{2} \sin \Phi\right) \wedge v_{3} \wedge\left(\frac{d b}{d s} v_{1}-\frac{d a}{d s} v_{2}\right) \\
& =\left(\cos \Phi \frac{d a}{d s}+\frac{d b}{d s} \sin \Phi\right) v_{1} \wedge v_{2} \wedge v_{3}
\end{aligned}
$$

Developing $d a, d b$ on $\omega_{1}, \omega_{2}$ as above, we get

$$
\begin{aligned}
\tau_{r}(s) & =\left[\begin{array}{c}
\cos \Phi\left(L_{12} \cos \Phi+L_{22} \sin \Phi\right) \\
-\sin \Phi\left(L_{11} \cos \Phi+L_{12} \sin \Phi\right)
\end{array}\right] \\
& =\left[L_{12} \cos 2 \Phi+\frac{\sin 2 \Phi}{2}\left(L_{22}-L_{11}\right)\right] .
\end{aligned}
$$


The curvature lines are defined by $\tau_{r}(s)=0$, hence

$$
\tan 2 \Phi=\frac{2 L_{12}}{L_{11}-L_{22}}
$$

as we have already obtained previously in Eq. (219).

Geodesics The geodesic curvature $\rho_{g}(s)$ is given by

$$
\begin{aligned}
I \rho_{g}(s)= & \frac{d(O M)}{d s} \wedge \frac{d^{2}(O M)}{d s^{2}} \wedge v_{3} \\
= & \frac{1}{d s}\left(v_{1} \cos \Phi+v_{2} \sin \Phi\right) \\
& \wedge\left[\begin{array}{c}
(d \Phi+d c)\left(-v_{1} \sin \Phi+v_{2} \cos \Phi\right) \\
+v_{3}(\sin \Phi d a-\cos \Phi d b)
\end{array}\right] \wedge v_{3} \\
= & \left(\frac{d c}{d s}+\frac{d \Phi}{d s}\right) I .
\end{aligned}
$$

The geodesic lines correspond to $\rho_{g}(s)=0$ and thus to $d c+d \Phi=0$ or equivalently

$$
c_{1} d u+c_{2} d v+d\left(\operatorname{Arctan} \frac{\omega_{2}}{\omega_{1}}\right)=0 .
$$

Hence, the equation of the geodesic is given by [15, II, p. 414]

$$
c_{1} d u+c_{2} d v+d\left(\operatorname{Arctan} \frac{A_{2} d u+B_{2} d v}{A_{1} d u+B_{1} d v}\right)=0
$$

where $c_{1}, c_{2}$ are expressed by Eqs. $(188,189)$.

\subsection{Example}

Consider as surface the sphere of radius $r, x(t)=(r \sin \theta \cos \varphi) I i+(r \sin \theta \sin \varphi) I j+$ $(r \cos \theta) I k$. One has $d x=\omega_{1} v_{1}+\omega_{2} v_{2}$ with $\omega_{1}=r d \theta, \omega_{1}=r \sin \theta d \varphi\left(A_{1}=\right.$ $\left.r, B_{1}=0, A_{2}=0, B_{2}=r \sin \theta\right)$ and

$$
\begin{aligned}
& v_{1}=(\cos \theta \cos \varphi) I i+(\cos \theta \sin \varphi) I j-(\sin \theta) I k \\
& v_{2}=-(\sin \varphi) I i+(\cos \varphi) I j \\
& v_{3}=\left(v_{1} \wedge v_{2}\right)^{*}=(\sin \theta \cos \varphi) I i+(\sin \theta \sin \varphi) I j+(\cos \theta) I k .
\end{aligned}
$$

This frame $\left(v_{i}=r e_{i} r_{c}\right)$ is obtained from the canonical basis $e_{i}$ via a rotation $r_{1}$ of $\varphi$ around $e_{3}$, followed by a rotation $r_{2}$ of $\theta$ around the axis $r_{1} e_{2} r_{1 c}$, hence,

$$
\begin{aligned}
r= & r_{1} r_{2}=e^{k \frac{\varphi}{2}} e^{j \frac{\theta}{2}} \\
= & \cos \frac{\theta}{2} \cos \frac{\varphi}{2}-\left(\sin \frac{\theta}{2} \sin \frac{\varphi}{2}\right) i \\
& +\left(\sin \frac{\theta}{2} \cos \frac{\varphi}{2}\right) j+\left(\cos \frac{\theta}{2} \sin \frac{\varphi}{2}\right) k .
\end{aligned}
$$


The affine connection bivector $d \iota=2 r_{c} d r$ is

$$
\begin{aligned}
d \iota & =-(d \varphi \sin \theta) i+(d \theta) j+(d \varphi \cos \theta) k \\
& =(d a) i+(d b) j+(d c) k,
\end{aligned}
$$

leading to (with $d a=a_{1} d \theta+a_{2} d \varphi$, etc.)

$$
\begin{aligned}
& a_{1}=0, a_{2}=\sin \theta, b_{1}=1, b_{2}=0 \\
& c_{1}=0, c_{2}=\cos \theta .
\end{aligned}
$$

The Gaussian and mean curvature are respectively

$$
\begin{aligned}
& K=\frac{a_{1} b_{2}-a_{2} b_{1}}{A_{1} B_{2}-A_{2} B_{1}}=\frac{1}{r^{2}} \\
& H=\frac{-b_{1} B_{2}+b_{2} A_{2}+a_{2} A_{1}-a_{1} B_{1}}{2\left(A_{1} B_{2}-A_{2} B_{1}\right)}=-\frac{1}{r} .
\end{aligned}
$$

\section{Conclusion}

The paper has presented a biquaternion calculus, having an associative exterior product, and shown how differential geometry can be expressed within this new algebraic framework. The method presented here can be extended to other spaces such as a pseudo-Euclidean $4 \mathrm{D}$ space. It is hoped that this paper will further interest in these new algebraic tools and provide new perspectives for geometric modeling.

Acknowledgments. This work was conducted in the framework of LabEx Celya and PRIMES (Physics Radiobiology Medical Imaging and Simulation).

\section{References}

1. Gürlebeck, K., Sprössig, W.: Quaternionic and Clifford Calculus for Physicists and Engineers. Wiley, New York (1997)

2. Vince, J.: Geometric Algebra for Computer Graphics. Springer, London (2008)

3. SNYGG, J.: A New Approach to Differential Geometry using Clifford's Geometric Algebra. Springer, New York (2012).

4. Casanova, G.: L'algèbre vectorielle. PUF, Paris (1976)

5. Girard, P.R., Pujol, R., Clarysse, P., Marion, A., Goutte, R., Delachartre, P.: Analytic Video (2D+t) Signals by Clifford Fourier Transforms in Multiquaternion Grassmann-Hamilton-Clifford Algebras. In Quaternion and Clifford Fourier Transforms and Wavelets, E. Hitzer, S. J. Sangwine, eds., pp. 197-219. Birkhäuser, Basel (2013)

6. GIRARD, P.R.: Quaternion Grassmann-Hamilton-Clifford-algebras: new mathematical tools for classical and relativistic modeling. In O. Dössel and W. C. Schlegel (Eds), World Congress 2009, IFMBE Proceedings, vol 25/IV, 65-68 (2009). 
7. Girard, P.R.: Quaternions, Clifford Algebras and Relativistic Physics. Birkhäuser, Basel (2007)

8. Girard, P.R.: Quaternions, Algèbre de Clifford et Physique Relativiste. PPUR, Lausanne (2004)

9. Girard, P.R.: Quaternions, Clifford Algebra and Symmetry Groups. In Applications of Geometric Algebra in Computer Science and Engineering, L. Dorst, C. Doran and J. Lasenby, eds.. Birkhäuser, Boston, pp. 307-315 (2002)

10. Girard, P.R.: Einstein's equations and Clifford algebra. Advances in Applied Clifford Algebras, vol. 9, 2, pp. 225-230 (1999)

11. GiRARD, P.R.: The quaternion group and modern physics. Eur. J. Phys., vol. 5, pp. 25-32 (1984)

12. Gsponer, A., Hurni, J.P.: Quaternions in mathematical physics (1): Alphabetical bibliography, math-ph, 0510059V4, (1430 references). Published in the internet at http://www.arxiv.org/abs/arXiv:mathph/0510059 (2008)

13. Gsponer, A., Hurni, J.P.: Quaternions in mathematical physics (2): Analytical bibliography, math-ph, 0511092V3, (1100 references). Published in the internet at http://www.arxiv.org/abs/arXiv:mathphy/0511092 (2008)

14. Hamilton, W.R.: The Mathematical Papers. Ed. A. W. Conway for the Royal Irish Academy, Cambridge University Press, Cambridge (1931-67)

15. Hamilton, W.R.: Elements of Quaternions. 2 vols (1899-1901), reprinted 1969, Chelsea, New York (1969)

16. Hankins, T.L.: Sir William Rowan Hamilton. Johns Hopkins University Press, Baltimore (1980)

17. Crowe, M.J.: A History of Vector analysis: The Evolution of the Idea of a Vectorial System. University of Notre Dame, Notre Dame, London (1967)

18. Grassmann, H.: Die lineale Ausdehungslehre: ein neuer Zweig der Mathematik, dargestellt und durch Anwendungen auf die übrigen Zweige der Mathematik, wie auch die Statik, Mechanik, die Lehre von Magnetismus und der Krystallonomie erläutert. Wigand, Leipzig, 1844. Second ed. (1878)

19. Grassmann, H.: Mathematische und physikalische Werke. F. Engel, ed., 3 vols. in 6 pts, Leipzig (1894-1911)

20. Grassmann, H.: Der Ort der Hamilton'schen Quaternionen in der Ausdehnungslehre. Math. Ann., vol. 12, 375-386 (1877).

21. Petsche, H.-J.: Grassmann. Birkhäuser, Basel (2006)

22. Clifford, W.K.: Applications of Grassmann's extensive algebra. J. Math., 1, 350358 (1878).

23. Clifford, W.K.: Mathematical Papers. Ed. R. Tucker (1882), reprinted Chelsea, New-York, pp. 266-276 (1968)

24. Lagally, M.: Vorlesungen über Vektorrechnung. 5th ed., Akademische Verlagsgesellschaft, Leipzig (1956)

25. Fenr, H.: Application de la Méthode Vectorielle de Grassmann à la Géométrie Infinitésimale. Carré et Naud, Paris (1899)

26. Guggenheimer, H.W.: Differential Geometry. Dover, New York (1976) 
A Representation of biquaternions by $4 \times 4$ real matrices

$$
\begin{gathered}
e_{1}=I i=\left[\begin{array}{cccc}
0 & 1 & 0 & 0 \\
1 & 0 & 0 & 0 \\
0 & 0 & 0 & -1 \\
0 & 0 & -1 & 0
\end{array}\right], e_{2}=I j=\left[\begin{array}{cccc}
-1 & 0 & 0 & 0 \\
0 & 1 & 0 & 0 \\
0 & 0 & -1 & 0 \\
0 & 0 & 0 & 1
\end{array}\right] \\
e_{3}=I k=\left[\begin{array}{cccc}
0 & 0 & 0 & -1 \\
0 & 0 & -1 & 0 \\
0 & -1 & 0 & 0 \\
-1 & 0 & 0 & 0
\end{array}\right], e_{3} e_{2}=i=\left[\begin{array}{cccc}
0 & 0 & 0 & -1 \\
0 & 0 & 1 & 0 \\
0 & -1 & 0 & 0 \\
1 & 0 & 0 & 0
\end{array}\right] \\
e_{1} e_{3}=j=\left[\begin{array}{cccc}
0 & 0 & -1 & 0 \\
0 & 0 & 0 & -1 \\
1 & 0 & 0 & 0 \\
0 & 1 & 0 & 0
\end{array}\right], e_{2} e_{3}=k=\left[\begin{array}{cccc}
0 & -1 & 0 & 0 \\
1 & 0 & 0 & 0 \\
0 & 0 & 0 & 1 \\
0 & 0 & -1 & 0
\end{array}\right] \\
e_{1} e_{2} e_{3}=I=\left[\begin{array}{cccc}
0 & 0 & -1 & 0 \\
0 & 0 & 0 & 1 \\
1 & 0 & 0 & 0 \\
0 & -1 & 0 & 0
\end{array}\right], 1=\left[\begin{array}{cccc}
1 & 0 & 0 & 0 \\
0 & 1 & 0 & 0 \\
0 & 0 & 1 & 0 \\
0 & 0 & 0 & 1
\end{array}\right]
\end{gathered}
$$

B Representation of biquaternions by $2 \times 2$ complex Pauli matrices

$\left(i^{\prime}\right.$ : ordinary complex imaginary)

$$
\begin{aligned}
e_{1} & =I i=\sigma_{1}=\left[\begin{array}{ll}
0 & 1 \\
1 & 0
\end{array}\right], e_{2}=I j=\sigma_{2}=\left[\begin{array}{cc}
0 & -i^{\prime} \\
i^{\prime} & 0
\end{array}\right] \\
e_{3} & =I k=\sigma_{1}=\left[\begin{array}{ll}
1 & 0 \\
0 & -1
\end{array}\right], e_{3} e_{2}=i=\left[\begin{array}{cc}
0 & -i^{\prime} \\
-i^{\prime} & 0
\end{array}\right] \\
e_{1} e_{3} & =j=\left[\begin{array}{ll}
0 & -1 \\
1 & 0
\end{array}\right], e_{2} e_{3}=k=\left[\begin{array}{cc}
-i^{\prime} & 0 \\
0 & i^{\prime}
\end{array}\right] \\
e_{1} e_{2} e_{3} & =I=\left[\begin{array}{ll}
i & 0 \\
0 & i
\end{array}\right], 1=\left[\begin{array}{ll}
1 & 0 \\
0 & 1
\end{array}\right] .
\end{aligned}
$$

\section{Work-sheet: biquaternions (Mathematica)}

$<<$ Quaternions

( $*$ product of two biquaternions $a=a_{1}+I a_{2}, b=b_{1}+I b_{2}, a_{i}, b_{i} \in \mathbb{H} ;$ a double star $* *$ means a quaternion product $*)$

$$
\begin{aligned}
C P\left[a_{-}, b_{-}\right]: & =\{(a[[1]] * * b[[1]])-(a[[2]] * * b[[2]]), \\
& (a[[2]] * * b[[1]])+(a[[1]] * * b[[2]])\}
\end{aligned}
$$


$\left({ }^{*}\right.$ conjugate $\left.K^{*}\right)$

$$
\begin{gathered}
K\left[a_{-}\right]:=\{\text {Quaternion }[a[[1,1]],-a[[1,2]],-a[[1,3]],-a[[1,4]]], \\
\text { Quaternion }[a[[2,1]],-a[[2,2]],-a[[2,3]],-a[[2,4]]]\}
\end{gathered}
$$

(*sum and difference*)

$$
\begin{aligned}
\operatorname{csum}\left[a_{-}, b_{-}\right] & :=\{a[[1]]+b[[1]], a[[2]]+b[[2]]\} \\
\operatorname{cdif}\left[a_{-}, b_{-}\right]: & =\{a[[1]]-b[[1]], a[[2]]-b[[2]]\}
\end{aligned}
$$

$(*$ multiplication by a scalar*)

$$
f \operatorname{clif}\left[f_{-}, a_{-}\right]:=\{f * a[[1]], f * a[[2]]\}
$$

$\left(*\right.$ products $\left.\frac{1}{2}(a b+b a), \frac{1}{2}(a b-b a) *\right)$

$$
\begin{aligned}
& \operatorname{int}\left[a_{-}, b_{-}\right]:=\{\operatorname{fclif}[1 / 2, \operatorname{csum}[C P[a, b], C P[b, a]]]\} \\
& \operatorname{ext}\left[a_{-}, b_{-}\right]:=\{\operatorname{fclif}[1 / 2, \operatorname{cdif}[C P[a, b], C P[b, a]]]\}
\end{aligned}
$$

$\left(*\right.$ products $\left.-\frac{1}{2}(a b+b a),-\frac{1}{2}(a b-b a) *\right)$

$$
\begin{aligned}
& \operatorname{mint}\left[a_{-}, b_{-}\right]:=\{\operatorname{fclif}[-1 / 2, \operatorname{csum}[C P[a, b], C P[b, a]]]\} \\
& \operatorname{mext}\left[a_{-}, b_{-}\right]:=\{\operatorname{fclif}[-1 / 2, \operatorname{cdif}[C P[a, b], C P[b, a]]]\}
\end{aligned}
$$

(*example: product of two biquaternions $A$ and $B, w=A B *$ )

$$
\begin{aligned}
& A=\{\text { Quaternion }[1,3,0,4], \text { Quaternion }[2,1,5,1]\} \\
& B=\{\text { Quaternion }[1,7,8,1], \text { Quaternion }[2,1,0,1]\} \\
& w=\operatorname{Simplify}[C P[A, B]]
\end{aligned}
$$

$(*$ result $*)$

$\{$ Quaternion $[-26,-31,23,30]$, Quaternion $[-51,19,28,-15]\}$ 